Artigo / Article

\title{
Mieloma Múltiplo e distúrbios da hemostasia
}

\section{Hemostatic abnormalities in Multiple Myeloma}

Elbio A. D'Amico ${ }^{1,2}$

Paula R. Villaça $a^{1,2}$

\begin{abstract}
O mieloma múltiplo (MM) é o tumor de células plasmocitárias que corresponde a aproximadamente $10 \%$ das neoplasias hematológicas. Durante o seu curso clínico, relata-se que $15-30 \%$ dos pacientes podem apresentar manifestações hemorrágicas, decorrentes de vários mecanismos fisiopatológicos. Até 1999, era descrito que os pacientes com MM ativo apresentavam incidência de até $10 \%$ de tromboembolismo. Porém, com a introdução da talidomida no arsenal terapêutico do MM, foi demonstrado aumento importante da freqüência dos eventos trombóticos venosos e arteriais, especialmente quando associada com outros medicamentos, alcançando a taxa de $35 \%$. Várias medidas tromboprofiláticas farmacológicas foram empregadas visando reduzir essas intercorrências, com resultados variáveis. Até o presente momento ainda não se demonstrou qual o regime antitrombótico mais adequado. Recentemente, uma pesquisa entre membros do Grupo Internacional de Trabalho sobre Mieloma (IMWG) resultou em algumas recomendações terapêuticas. Esta revisão sobre as alterações hemostáticas observadas no mieloma múltiplo procurou abordar os diferentes mecanismos responsáveis pelas hemorragias e tromboses observados no MM, as medidas tromboprofiláticas farmacológicas descritas e seus resultados, e as recentes recomendações do IMWG. Rev. bras. hematol. hemoter. 2007;29(1):92-97.
\end{abstract}

Palavras-chave: Mieloma múltiplo; talidomida; tromboembolismo venoso; tromboprofilaxia; heparina de baixo peso molecular; aspirina; varfarina.

\section{Introdução}

O mieloma múltiplo (MM) é o tumor de células plasmocitárias que corresponde a aproximadamente $10 \%$ das neoplasias hematológicas. Até os dias de hoje é considerado como uma doença incurável, mesmo após a introdução de algumas medidas terapêuticas que aumentaram a taxa de remissão completa, os indicadores de eventos livres de doença e a sobrevida global. ${ }^{1}$

As descrições clássicas relatam que, durante o curso clínico do MM, 15\%-30\% dos pacientes podem apresentar manifestações hemorrágicas, que variam de acordo com o tipo de MM (15\% para IgG e 30\% para IgA). ${ }^{2}$ São descritas várias causas como responsáveis por esses sangramentos, podendo acometer todos os componentes da hemostasia. Assim, são descritas: a) infiltração vascular pela célula ma- ligna, b) plaquetopenia secundária à infiltração medular ou ao tratamento realizado, c) anormalidades funcionais das plaquetas (incluindo uremia), d) doença ou síndrome de von Willebrand adquirida, e) deficiências adquiridas de fatores da coagulação secundárias à doença hepática, f) presença de anticoagulantes circulantes do tipo heparina e g) inibição da polimerização da fibrina. Outras importantes condições associadas às manifestações hemorrágicas são a síndrome de hiperviscosidade e a sepse. ${ }^{3}$

As neoplasias são importantes fatores de risco para o desenvolvimento de eventos trombóticos, uma associação descrita por Armand Trousseau em 1865. O mieloma múltiplo não foge dessa regra. Até 1999 relatava-se que os pacientes com MM ativo, com necessidade de tratamento, apresentavam $10 \%$ de freqüência de tromboembolismo venoso. ${ }^{4,5} \mathrm{~A}$ partir desse ano, a talidomida foi incorporada ao arsenal

${ }^{1}$ Serviço de Hematologia do Hospital das Clínicas da Faculdade de Medicina da Universidade de São Paulo.

${ }^{2}$ Faculdade de Medicina da USP.

Correspondência: Elbio Antonio D’Amico

Rua Barata Ribeiro, 237 Conj.146 - Bela Vista

01308-000 - São Paulo-SP - Brasil

Tel.: (11) 3151-4354.

E-mail: elbio_damico@uol.com.br 
terapêutico empregado no tratamento do MM, resultando em aumento importante da taxa de resposta em pacientes previamente tratados. Contudo, isto também se associou a maior freqüência de TEV, especialmente quando a talidomida era administrada em associação com outros medicamentos, chegando a alcançar a taxa de $35 \% .{ }^{6}$ Dentre os eventos de TEV, os episódios de trombose venosa profunda são os mais observados, porém tromboses venosas em outras localizações e tromboses arteriais ${ }^{7}$ são também descritas nos pacientes sob uso da talidomida. Por esse motivo, várias medidas tromboprofiláticas passaram a ser empregadas.

\section{Talidomida e trombose}

O mecanismo pelo qual a talidomida, isoladamente ou associada a outros agentes tumorais, pode favorecer a trombose é desconhecido. Os eventos trombóticos não teriam relação com a dose utilizada de talidomida, geralmente ocorrem nos primeiros três meses de tratamento e apresentam maior freqüência quando a talidomida é usada em associação com quimioterápicos, particularmente doxorrubicina, e dexametasona, nos casos recém-diagnosticados, e, portanto, com maior massa tumoral, e nos pacientes que apresentam anormalidade no cromossomo 11. , $^{1,8,9,10}$ Porém, deve ser enfatizado que, nos pacientes com mieloma, freqüentemente estão presentes outros fatores de risco para TEV, como idade avançada, imobilização, aumento da viscosidade plasmática, uso de cateter venoso central e infecções associadas.

Foi sugerido que o efeito poderia envolver uma ação direta da talidomida sobre as células endoteliais previamente lesadas por agentes quimioterápicos, como a doxorrubicina. ${ }^{11}$ Zangari $\mathrm{M}$ et al $^{12}$ demonstraram maior risco trombótico em pacientes com resistência adquirida à proteína $\mathrm{C}$ ativada. Weber $\mathrm{D}$ et al desenvolveram trabalho avaliando fatores prótrombóticos antes e após o tratamento com talidomida. Mostraram que no período anterior à terapia específica havia aumento das concentrações de homocisteína em $56 \%$ dos pacientes e redução da antitrombina (AT), proteína C e proteína S em $42 \%, 18 \%$ e $12 \%$ deles, respectivamente. Além disso, $49 \%$ dos pacientes apresentaram a presença da mutação da enzima metileno-tetra-hidrofolato redutase termolábil (MTHFR) e 8\% deles tinham anticoagulante lúpico positivo. Com o tratamento, observou-se somente normalização dos níveis da AT. ${ }^{13}$ Em 2003, Ward CM et al ${ }^{14}$ relataram que os pacientes que evoluíam com aumento das concentrações de FVIII:C e do fator von Willebrand durante o tratamento com talidomida, e muitos dos que apresentaram eventos de TEV durante o tratamento, já apresentavam níveis basais elevados do FVIII:C e do fator von Willebrand, além de resistência à proteína $\mathrm{C}$ ativada. Porém, no mesmo ano, Minnema MC et al também mostraram elevação dos níveis do FVIII:C e do antígeno do fator von Willebrand, porém relacionando esses achados com o estado da doença e não com o uso da talidomida. ${ }^{6}$ Mais recentemente, Baz et al demonstraram não somente aumento do fator von Willebrand, mas também hiperagregabilidade plaquetária no $28^{\circ}$ dia de tratamento com talidomida, associada à doxorrubicina peguilada, vincristina e dexametasona. ${ }^{15}$

\section{Profilaxia do TEV em pacientes com mieloma múltiplo recebendo talidomida}

Diferentes medidas de tromboprofilaxia farmacológica já foram empregadas nos pacientes com mieloma sob tratamento com vários esquemas onde se associa a talidomida. Dessa maneira, a varfarina, as heparinas de baixo peso molecular e a aspirina já foram empregadas como medidas profiláticas, refletindo o conhecimento limitado sobre a fisiopatologia da trombose relacionada com a talidomida, sendo que algumas vezes seus resultados foram comparados com aqueles obtidos sem o emprego de qualquer tromboprofilaxia. Porém, como será visto, ainda está para ser demonstrado qual o regime antitrombótico é mais eficaz para os pacientes com mieloma múltiplo recebendo tratamento com talidomida.

\section{Varfarina como tromboprofilaxia farmacológica}

Em 2003, Weber et al $^{16}$ trataram quarenta pacientes com diagnóstico recente de mieloma múltiplo sintomático com dexametasona e talidomida, pelo período mínimo de três meses. Os primeiros 24 pacientes receberam $1 \mathrm{mg} /$ dia de varfarina, como tromboprofilaxia. Como nesse grupo foram observados $25 \%$ de eventos de tromboembolismo venoso (TEV), os 16 pacientes seguintes fizeram profilaxia antitrombótica com dose terapêutica de varfarina (INR=2-3), resultando na ausência de TEV. Cavo et $a l^{17}$ empregaram o mesmo esquema terapêutico, por aproximadamente quatro meses, em 71 pacientes com diagnóstico de mieloma múltiplo, com o intuito de reduzir a massa tumoral antes da coleta de células CD $34^{+}$do sangue periférico e subseqüente transplante autólogo. Os primeiros 19 pacientes mostraram 26,3\% de episódios de trombose venosa profunda (TVP), o que originou mudança do protocolo, com a adição de varfarina em dose fixa diária de $1,25 \mathrm{mg}$, o que não causou alterações no INR. Os autores puderam observar redução dos eventos trombóticos (TVP) para $13,5 \%$, sendo que dois deles ocorreram dez e trinta dias após a suspensão do anticoagulante oral. Zangari et $a l^{18}$ distribuíram de maneira aleatória 256 pacientes com diagnóstico recente de mieloma múltiplo para tratamento citotóxico idêntico, com e sem talidomida. A fase de indução desse protocolo poliquimioterápico correspondia a um ciclo de VAD (vincristina, doxorrubicina e dexametasona), um ciclo de DCEP (dexametasona, ciclofosfamida, etoposide e cisplatina), um ciclo de CAD (ciclofosfamida, doxorrubicina e dexametasona) e um segundo ciclo de DCEP. Desses 256 pacientes, 221 não fizeram profilaxia antitrombótica (quimioterapia - QT- em 134 pacientes e quimioterapia + talidomida - QT+T - em 87) e 35 receberam tratamento quimioterápico + talidomida associado à varfarina (QT+T+varfarina) em baixa dose (1mg/dia). Os 
autores observaram que no grupo QT a freqüência de trombose venosa profunda foi de $14,18 \%$, enquanto nos grupos $\mathrm{QT}+\mathrm{T}$ e QT+ T+ varfarina as freqüências de TVP foram de 34,5\% e 31,4\%, respectivamente. Chanan-Khan et al ${ }^{19}$ empregaram o protocolo VAD-talidomida em $16 \mathrm{pa}$ cientes com mieloma múltiplo recém-diagnosticado, usando varfarina, na dose de 1-2 mg como profilaxia antitrombótica, observando $12 \%$ de trombose venosa profunda. Em 2005, Cavo et al ${ }^{20}$ relataram os resultados do estudo expandido iniciado em 2004. Neste trabalho, cem pacientes com mieloma sintomático foram tratados com o protocolo dexametasona-talidomida, como terapia primária preparatória para o transplante autólogo com célulastronco periféricas $\left(\mathrm{CD} 34^{+}\right)$. Nos primeiros 19 pacientes, que não fizeram profilaxia antitrombótica, a freqüência de trombose venosa profunda foi de $26 \%$, enquanto nos 81 subseqüentes, que receberam varfarina na dose fixa de 1,25 mg por dia, a freqüência de TVP foi de $12 \%$. Com a introdução da doxorrubicina lipossomal, foram utilizados protocolos associando quimioterápico com a dexametasona e talidomida, em doses baixas (100 mg diariamente), visando observar sua resposta terapêutica em pacientes com mieloma avançado. Nos pacientes $(n=50)$ onde se incluiu a varfarina como medida tromboprofilática, na dose diária de 1,25 mg, observaram-se 12\% de eventos de tromboembolismo venoso, sendo $10 \%$ de trombose venosa profunda (5/50) e $2 \%$ de embolia pulmonar (1/50). ${ }^{21}$

Recentemente foram relatados os resultados da profilaxia antitrombótica com varfarina, em doses variáveis de acordo com o peso dos pacientes $(<70 \mathrm{~kg}=1 \mathrm{mg} / \mathrm{dia},>70 \mathrm{~kg}=$ $2 \mathrm{mg} /$ dia) em indivíduos com diagnóstico de mieloma múltiplo $(n=80)$, tratados com diferentes esquemas terapêuticos (talidomida isolada; dexametasona + talidomida; bortezomib + doxorrubicina lipossomal + talidomida, VAD + talidomida; bortezomib + ciclofosfamida + dexametasona + talidomida), obtendo-se a incidência de 6,25\% de eventos de tromboembolismo venoso..$^{22}$ (Tabela 1 )

\section{Heparina de baixo peso molecular como medida tromboprofilática}

A nadroparina, 2.850 IE anti-Xa ou 5.700 IE anti-Xa, para os pacientes com peso superior a 90 quilos, foi utilizada por Minnema et $a l^{23}$ em 211 pacientes com diagnóstico recente de mieloma múltiplo, tratados com três ciclos de talidomida, doxorrubicina e dexametasona (TAD), seguidos de coleta de células-tronco periféricas, após mobilização com ciclofosfamida, doxorrubicina, dexametasona e G-CSF. Após a terapia de indução, os pacientes recebiam 1-2 ciclos de altas doses de melfalano, com resgate com células-tronco autólogas. A profilaxia com nadroparina era iniciada no primeiro dia do primeiro ciclo e mantida até uma semana antes da quimioterapia para mobilização celular. Um total de 19 pacientes (9\%) apresentou eventos de tromboembolismo venoso, sendo que a maioria $(89,5 \%)$ deles ocorreu durante a terapia de indução, correspondendo principalmente à trombose venosa profunda de membros inferiores (82,3\%), mas também sendo descritos eventos de embolia pulmonar, associada ou não à TVP dos membros inferiores, e trombose venosa profunda de membro superior. Foi relatado um caso de TEV após a quimioterapia de mobilização celular e outro caso após o uso do melfalano.

A enoxaparina foi usada como profilaxia antitrombótica por alguns investigadores. Zangari et al ${ }^{18}$ empregaram dose diária de 40 mg em 68 pacientes com diagnóstico recente de mieloma múltiplo, tratados com o esquema poliquimioterápico associado à talidomida, já descrito anteriormente (varfarina como tromboprofilaxia farmacológica), observando a incidência de trombose venosa profunda de $14,7 \%$. O grupo italiano de estudo do mieloma múltiplo (GIMENA) publicou dois trabalhos onde a enoxaparina foi o agente tromboprofilático utlizado. No primeiro deles, ${ }^{24}$ a enoxaparina ( $40 \mathrm{mg} / \mathrm{dia}$ ) foi administrada durante os primeiros quatro ciclos de quimioterapia (melfalano, prednisona e talidomida), de um total de seis ciclos, em 64 pacientes com diagnóstico recente de mieloma múltiplo. Foram observados dois episódios de tromboembolismo venoso (3,12\%), que ocorreram dentro de dois 
meses após a suspensão da heparina de baixo peso molecular. Em 65 pacientes submetidos ao mesmo esquema quimioterápico, porém sem fazer tromboprofilaxia, a incidência de tromboembolismo venoso foi de $20 \%$, sendo que esses eventos aconteceram durante os primeiros quatro meses de tratamento, o que justificou o tempo empregado de tromboprofilaxia no primeiro grupo. O segundo trabalho do grupo italiano ${ }^{25}$ usou os mesmos protocolos de quimioterapia e de tromboprofilaxia (por quatro meses somente) em 78 pacientes, também com diagnóstico recente de mieloma múltiplo. Foram relatados quatro episódios de tromboembolismo venoso $(5,13 \%)$, sendo que dois deles ocorreram dois meses após a interrupção da enoxaparina.

Tabela 2. Incidência de tromboembolismo venoso (TEV) em pacientes com mieloma múltiplo em tratamento com talidomida, fazendo profilaxia antitrombótica com heparina de baixo peso molecular

\begin{tabular}{|c|c|c|c|c|c|}
\hline Autor & Tratamento & Diagnóstico & $\begin{array}{l}\text { Número de } \\
\text { pacientes }\end{array}$ & $\begin{array}{l}\text { Profilaxia } \\
\text { de TEV }\end{array}$ & $\begin{array}{c}\text { Incidência } \\
\text { de TEV } \\
(\%)\end{array}$ \\
\hline Minnema et $\mathrm{a}^{23}$ & $\begin{array}{l}\text { VAD } \\
\text { TAD }\end{array}$ & $\begin{array}{l}\text { DR } \\
\text { DR }\end{array}$ & $\begin{array}{l}201 \\
211\end{array}$ & $\begin{array}{c}\text { Ausente } \\
\text { Nadroparina } \\
2.850-5.700 \mathrm{IE} \\
\text { anti-Xa }\end{array}$ & $\begin{array}{l}5 \\
9\end{array}$ \\
\hline Zangari et $\mathrm{a}^{18}$ & $\begin{array}{c}\mathrm{QT} \\
\mathrm{QT+T}\end{array}$ & $\begin{array}{l}\text { DR } \\
\text { DR }\end{array}$ & $\begin{array}{l}62 \\
62\end{array}$ & $\begin{array}{c}\text { Ausente } \\
\text { Enoxaparina } \\
40 \mathrm{mg} / \mathrm{d}\end{array}$ & $\begin{array}{l}14,5 \\
14,7\end{array}$ \\
\hline Palumbo et al ${ }^{24}$ & MPT & DR & $\begin{array}{l}65 \\
64\end{array}$ & $\begin{array}{c}\text { Ausente } \\
\text { Enoxaparina } \\
40 \mathrm{mg} / \mathrm{d} / 4 \mathrm{meses}\end{array}$ & $\begin{array}{c}20 \\
3,12^{\S}\end{array}$ \\
\hline Palumbo et a $\left.\right|^{25}$ & MPT & DR & 78 & $\begin{array}{c}\text { Enoxaparina } \\
40 \mathrm{mg} / \mathrm{d} / 4 \text { meses }\end{array}$ & $5,13^{\S}$ \\
\hline Barlogie et $\mathrm{al}^{26}$ & $\mathrm{QT}+\mathrm{T} \mathrm{M}$ & & $\begin{array}{l}162 \\
152\end{array}$ & $\begin{array}{l}\text { Sem profilaxia } \\
\text { Com profilaxia }\end{array}$ & $\begin{array}{l}34 \\
24\end{array}$ \\
\hline
\end{tabular}

* $\mathrm{DR}=$ diagnóstico recente; $\mathrm{VAD}=$ vincristina, doxorrubicina, dexametasona;TAD= talidomida, doxorrubicina, dexametasona; $\mathrm{QT}=$ quimioterapia; $\mathrm{T}=$ talidomida; $\mathrm{MPT}=$ melfalano, prednisona, talidomida; $\mathrm{M}=$ melfalano; $\S$ após a suspensão da enoxaparina

Tabela 3. Incidência de tromboembolismo venoso (TEV) em pacientes com mieloma múltiplo em tratamento com talidomida, fazendo profilaxia antitrombótica com aspirina

\begin{tabular}{cccccc}
\hline Autor & Tratamento & Diagnóstico & $\begin{array}{c}\text { Número de } \\
\text { pacientes }\end{array}$ & $\begin{array}{c}\text { Profilaxia de } \\
\text { TEV }\end{array}$ & $\begin{array}{c}\text { Incidência } \\
\text { de TEV (\%) }\end{array}$ \\
\hline Hassoun et al $^{27}$ & AD $\rightarrow$ TD & RR & 31 & AAS $81 \mathrm{mg} / \mathrm{d}$ & 9,6 \\
Baz et al28 & DVd-T & DR e RR & 84 & AAS $81 \mathrm{mg} / \mathrm{d}$ & 17,8 \\
Hassoun et al ${ }^{29}$ & AD $\rightarrow$ TD & DR & 42 & AAS $81 \mathrm{mg} / \mathrm{d}$ & 11 \\
Hussein et al ${ }^{30}$ & DVd-T & DR e RR & 105 & AAS $81 \mathrm{mg} / \mathrm{d}$ & 25
\end{tabular}

* $\mathrm{DR}=$ diagnóstico recente; $\mathrm{RR}=$ recaída/resistência; $\mathrm{AD}=$ doxorrubicina, dexametasona; $\mathrm{TD}=$ talidomida + dexametasona; DVd-T= doxorrubicina peguilada, vincristina, dexametasona (freqüência reduzida), talidomida.
Barlogie et al, ${ }^{26}$ em 2006, publicaram sua experiência de tromboprofilaxia com heparina de baixo peso molecular, sem (emelas, em dose profilática, em pacientes com ememéticas. Comparando os 162 pacie não usaram a heparina de baixo peso molecular, com os 152 que utilizaram essa medida tromboprofilática, puderam observar redução da incidência de tromboembolismo venoso de $34 \%$ para $24 \%$. (Tabela 2 )

\section{Profilaxia antitrombótica com ácido acetil salicílico}

Em 2004, dois grupos diferentes publicaram suas observações iniciais sobre o uso do ácido acetil salicílico como tromboprofilaxia em pacientes com mieloma múltiplo sob tratamento com talidomida associada à quimioterapia. Hassoun et al ${ }^{27}$ relataram que, em 31 pacientes fazendo tratamento seqüencial com doxorrubicina e dexametasona, por três meses, e dexametasona e talidomida, por dois meses, e ácido acetil salicílico (81 mg/dia), a incidência de trombose venosa profunda foi de 9,6\%. Por sua vez, Baz et $a l^{28}$ trataram 103 pacientes com mieloma recém-diagnosticado ou em recaída/refratariedade, com o protocolo DVd-T (doxorrubicina peguilada, vincristina, dexametasona com freqüência reduzida e talidomida). Desse total de pacientes, 84 fizeram uso do ácido acetil salicílico, $81 \mathrm{mg}$ por dia. Observou-se que, nesse grupo, a incidência de trombose venosa profunda foi de $17,8 \%$, enquanto foi de $57,8 \%$ naqueles 19 que não utilizaram o AAS. Em 2005, Hassoun et $a l^{29}$ relataram os resultados expandidos do seu trabalho publicado no ano anterior. Em 42 pacientes com diagnóstico recente de mieloma empregaram o protocolo seqüencial AD (doxorrubicina + dexametasona), por 2-3 meses, e TD (talidomida + dexametasona), por dois meses, associando-se AAS (81 mg/d), desde o início do tratamento quimioterápico, observando a incidência de 4\% de embolia pulmonar e $7 \%$ de trombose venosa profunda. Em 2006, mais uma vez o grupo da Cleveland Clinic ${ }^{30}$ descreveu seus resultados referentes ao tratamento de 102 pacientes com mieloma múltiplo (53 recém-diagnosticados e 49 em recaída/ 
refratariedade) tratados com o protocolo DVd-T, associado à aspirina $(81 \mathrm{mg} / \mathrm{dia})$, mostrando $25 \%$ de eventos de tromboembolismo venoso. (Tabela 3)

O grande objetivo ao se empregarem medidas tromboprofiláticas farmacológicas nos pacientes com mieloma múltiplo sob tratamento com talidomida associada à dexametasona e / ou doxorrubicina é se alcançar uma incidência de episódios de tromboembolismo venoso que seja igual ou inferior à incidência que se obtinha anteriormente sem o uso da talidomida. Vários agentes tromboprofiláticos já foram avaliados, com resultados discrepantes entre os mesmos agentes e, até momento, sem estudos comparando os resultados das diferentes modalidades tromboprofiláticas empregadas.

O Grupo Internacional de Trabalho sobre o Mieloma (IMWG) fez recentemente algumas recomendações baseadas numa pesquisa feita entre seus membros. A maioria deles julgou que a terapia associada com altas doses de dexametasona, agentes alquilantes, doxorrubicina/doxorrubicina lipossomal e eritropoetina aumenta o risco de trombose venosa profunda relacionada com a terapia com talidomida ou lenalidomida. A aspirina (81 mg ou $325 \mathrm{mg}$ ) foi o método preferido de profilaxia, quando ela está indicada. Contudo, $65 \%$ dos membros que responderam à pesquisa (23) preferiram a varfarina em dose terapêutica ou uma heparina de baixo peso molecular quando a doxorrubicina/doxorrubicina lipossomal ou melfalano está associada ao regime terapêutico empregado. Quando se empregam os esquemas VAD, MP, bortezomib com ou sem dexametasona, não há necessidade de tromboprofilaxia. Todos estavam de acordo quanto à realização de trabalhos randomizados. ${ }^{31}$

Considerando-se ainda que o evento trombótico é o resultado da associação de condições trombogênicas presentes naquele momento, todos os fatores de risco para trombose devem ser avaliados e, quando necessário, corrigidos. Desde que possível, deve ser estimulado o emprego de medidas tromboprofiláticas mecânicas, como deambulação, fisioterapia de membros inferiores, compressão intermitente de membros inferiores e uso de meia elástica.

\section{Recomendações}

- Quando a talidomida é empregada isoladamente não há necessidade do uso de tromboprofilaxia farmacológica.

- A aspirina é a medicação antitrombótica mais indicada pelos membros do Grupo Internacional de Trabalho sobre o Mieloma (IWGM); porém, quando a talidomida é empregada em associação com doxorrubicina/doxorrubicina lipossomal ou melfalano, a varfarina em dose terapêutica (INR entre 2 e 3) ou uma heparina de baixo peso molecular são as opções mais recomendadas pelo IWGM.
- O uso da varfarina em doses terapêuticas implica o seu controle laboratorial rigoroso, visto sua associação com maior freqüência de sangramentos.

- Quando se empregam os esquemas VAD, MP, bortezomibe com ou sem dexametasona, não há necessidade de tromboprofilaxia medicamentosa.

- Sempre que possível, devem-se associar métodos mecânicos de profilaxia antitrombótica.

\section{Abstract}

Multiple myeloma (MM) is the tumor of plasma cells that accounts for approximately $10 \%$ of hematological malignancies. During the clinical course of MM, hemorrhagic symptoms are described in 15-30\% of patients, due to several causes. Until 1999, it was reported that up $10 \%$ of patients with active MM had venous thromboembolic events. Nevertheless, since the introduction of thalidomide in the therapeutic arsenal of MM, an important increase of the incidence of arterial and venous thrombotic events were described (up 35\%), especially when used in association with other drugs. Several pharmacological thromboprophylactic measures have been introduced in order to decrease these events, but with variable results. The most adequate antithrombotic regimen has not yet been demonstrated. Recently the International Myeloma Working Group (IMWG) developed a survey among its members, resulting in some therapeutic recommendations. This review about hemostatic abnormalities in multiple myeloma had the intention of discussing the different mechanisms responsible for hemorrhagic and thrombotic events in MM, the pharmacological thrombophophylactic measures reported, their results and the recent recommendations of the IMWG. Rev. bras. hematol. hemoter. 2007;29(1):92-97.

Key words: Multiple myeloma; thalidomide; venous thromboembolism; thromboprophylaxis; low molecular weight heparin; aspirin; warfarin.

\section{Referências Bibliográficas}

1. Barbui T, Falanga A. Thalidomide and thrombosis in multiple myeloma. J Thromb Haemost 2003;1:421-422.

2. Barlogie B. Plasma cell myeloma. In: Williams. Hematology. Beutler E, Lichtman MA, Coller BS, Kipps TJ, eds. 5 ed, McGraw-Hill, New York, 1995. p. 1.109-1.126.

3. Eby C, Blinder M. Hemostatic complications associated with paraproteinemias. Curr Hematol Rep 2003;2:388-394.

4. Barlogie B, Jagannath S, Desikan KR, Mattox S, Vesole D, Siegel D, et al. Total therapy with tandem transplants for newly diagnosed multiple myeloma. Blood 1999;93:55-65.

5. Srkalovic G, Cameron MG, Rybicki L, Deitcher SR, Kattke-Marchant $\mathrm{K}$, Hussein MA. Monoclonal gammopathy of undetermined significance and multiple myeloma are associated with an increased incidence of venothromboembolic disease. Câncer 2004; 101:558-566. 
6. Minnema MC, Fijnheer R, De Groot PG, Lokhorst HM. Extremely high levels of von Willebrand factor antigen and of procoagulant factor VIII found in multiple myeloma patients are associated with activity status but not with thalidomide treatment. J Thromb Haemost 2003;1:445-449.

7. Scarpace SL, Hahn T, Roy H, Brown K, Paplham P, Chanan-Khan A, et al. Arterial thrombosis in four patients treated with thalidomide. Leuk Lymphoma 2005;46:239-242.

8. Rodeghiero F, Elice F. Thalidomide and thrombosis. Pathophysiol Haemost Thromb 2003;33(suppl 1):15-18.

9. Husseim MA. Thromboembolism risk reduction in multiple myeloma patients treated with immunomodulatory drug combinations. Thromb Haemost 2006;95:924-930.

10. Rus C, Bazzan M, Palumbo A, Bringhen S, Boccadoro M. Thalidomide in front line treatment in multiple myeloma: serious risk of venous thromboembolism and evidence for thromboprophylaxis. J Thromb Haemost 2004;2:2.063-2.065.

11. Kaushal V, Kaushal GP, Anaissie EJ, Kurylo P, Kohli M, Fink LM, et al. Thalidomide-induced coagulopathy: evidence for an indirect effect on endothelium. Blood 2002;100:3.771 (abstract).

12. Zangari M, Saghafifar F, Anaissie E, Badros A, Desikan R, Fassas A, et al. Activated protein $\mathrm{C}$ resistance in the absence of factor Leiden is a common finding in multiple myeloma and is associated with an increased risk of thrombotic complications. Blood Coagul Fibrinolysis 2002;13:187-192.

13. Weber D, Ginsberg C, Walker P, Oholendt M, Rankin K, Gavino $M$, et al. Correlation of thrombotic/embolic events (T/E) with features of hypercoagulability in previously untreated patients before and after treatment with thalidomide $(\mathrm{T})$ and thalidomidedexamethasone (TD). Blood 2002;100:787 (abstract).

14. Ward CM, Yen T, Harvie R, Pavlakis N. Elevated levels of factor VIII and von Willebrand factor after treatment for malignancy: relationship to thromboembolic events. Hematol J 2003; 4 (suppl 1):365(abstract).

15. Baz R, Li L, Kottke-Marchant K, Srkalovic G, McGowan B, Yiannaki $\mathrm{E}$, et al. The role of aspirin in the prevention of thrombotic complications of thalidomide and anthracycline-based chemotherapy for multiple myeloma. Mayo Clin Proc 2005; 80:1.568-1.574.

16. Weber D, Rankin K, Gavino M, Delasalle K, Alexanian R. Thalidomide alone or with dexamethasone for previously untreated multiple myeloma. J Clin Oncol 2003;21:16-19.

17. Cavo M, Zamagni E, Tosi P, Cellini C, Cangini D, Tacchetti P, et al. First-line therapy with thalidomide and dexamethasone in preparation for autologous stem cell transplantation for multiple myeloma. Haematologica 2004;89:826-831.

18. Zangari M, Barlogie B, Anaissie E, Saghafifar F, Eddlemon P, Jacobson J, et al. Deep vein thrombosis in patients with multiple myeloma treated with thalidomide and chemotherapy: effects of prophylactic and therapeutic anticoagulation. Brit J Haematol 2004;126:715-721.

19. Chanan-Khan AA, Miller KC, McCarthy P, Koryzna A, Kuoides P, Donohue $\mathrm{K}$, et al. VAD-t (vincristine, adriamycin, dexamethasone and low-dose thalidomide) is an effective initial therapy with high response rates for patients with treatment naïve multiple myeloma (MM). Blood 2004;104:abstract 3463.

20. Cavo M, Zamagni E, Tosi P, Tacchetti P, Cellini C, Cangini D, et al. Superiority of thalidomide and dexamethasone over vincristinedoxorubicin-dexamethasone (VAD) as primary therapy in preparation for autologous transplantation for multiple myeloma. Blood 2005;106:35-39.

21. Offidani M, Corvatta L, Marconi M, Visani G, Alesiani F, Brunori $\mathrm{M}$, et al. Low-dose thalidomide with pegylated liposomal doxorubicin and high-dose dexamethasone for relapsed/refractory multiple myeloma: a prospective, multicenter, phase II study. Haematologica 2006;91:133-136.

22. Miller KC, Manochakian R, Padmanabhan S, Coignet J-G, Musial L, DePaolo D, et al. Weight adjusted low-dose warfarin decreases the incidence of thalidomide $(\mathrm{T})$ associated venous thromboembolism (VTE) in patients (pts) with multiple myeloma (MM) and Waldestrom's macroglobulimenia (WM). Blood 2006;108: abstract 4101.

23. Minnema MC, Breitkreutz I, Auwerda JJA, van der Holt B, Cremer FW, van Marion AMW, et al. Prevention of venous thromboembolism with low molecular-weight heparin in patients with multiple myeloma treated with thalidomide and chemotherapy. Leukemia 2004;18:2.044-2.046.

24. Palumbo A, Brighen S, Caravita T, Merla E, Capparella V, Callea V, et al, for the Italian Multiple Myeloma Network, GIMENA. Oral melphalan and prednisone chemotherapy plus thalidomide compared with melphalan and prednisone alone in elderly patients with multiple myeloma: randomised controlled trial. Lancet 2006; 367:825-831.

25. Palumbo A, Rus C, Zeldis JB, Rodeghiero F, Boccadoro M, for the Italian Multiple Myeloma Network, GIMENA. Enoxaparin or aspirin for the prevention of recurrent thromboembolism in newly diagnosed myeloma patients treated with melphalan and prednisone plus thalidomide or lenalidomide. J Thromb Haemost 2006;4: 1.842-1.845.

26. Barlogie B, Tricot G, Anaissie E, Shaughnessy J, Rasmussen E, van Rhee F, et al. Thalidomide and hematopoietic-cell transplantation for multiple myeloma. N Engl J Med 2006;354:1.021-1.030.

27. Hassoun H, Reich L, Klimek VM, Kewalramani T, Dhodapkar M, Drake L, et al. Doxorubicin and dexamethasone followed by thalidomide and dexamethasone (AD-TD) as initial therapy for symptomatic patients with multiple myeloma. Blood 2004;104: abstract 2409.

28. Baz R, Marchant K, Yiannaki EO, Platt L, Brand C, Tso E, et al. Aspirin decreases the thrombotic complications (DVT) of liposomal doxorubicin and thalidomide (DVd-T) treatment of multiple myeloma (MM). Blood 2004;104; abstract 2397.

29. Hassoun H, Reich L, Klimek VM, Dhodapkar M, Cohen A, Kewalramani $\mathrm{T}$, et al. Doxorubicin and dexamethasone followed by thalidomide and dexamethasone is an effective well tolerate initial therapy for multiple myeloma. Brit J Haematol 2005;132: 155-161.

30. Hussein MA, Baz R, Srkalovic G, Agrawal N, Suppiah R, Hsi E, et al. Phase 2 study of pegylated liposomal doxorubicin, vincristine, decreased-frequency dexamethasone, and thalidomide in newly diagnosed and relapsed-refractory multiple myeloma. Mayo Clin Proc 2006;81:889-895.

31. Durie BGM, Richardson P, Palumbo A, Dimopoulos MA, Cavo M, Hajek R, et al. Deep vein thrombosis in myeloma: estimate of prevalence and recommendations for therapy based upon a survey of members of the International Myeloma Working Group (IMWG). Blood 2006;108:abstract 3571.

O tema apresentado e o convite ao(s) autor (es) consta da pauta elaborada pelo co-editor.

Avaliação: Co-editor e um revisor externo.

Publicado após revisão e concordância do editor.

Conflito de interesse: não declarado.

Recebido: 25/11/2006

Aceito: 15/01/2007 